\title{
Prediction of Buoyancy-Driven Flow in a Slot with Different Biot Numbers
}

\author{
Emel Selamet and Ahmet Selamet
}

\begin{abstract}
The projection method involving a Godunov type discretization for convective terms is applied to natural convection in water within a vertical slot. The variation of Nusselt number is examined for a range of Rayleigh numbers by imposing elevated temperature on the left wall and allowing convective heat transfer on the right. The results illustrate that heat transfer increases asymptotically with increasing Biot number. The rate of increase is a function of Rayleigh number and becomes more significant at higher Rayleigh numbers, as expected.
\end{abstract}

Index Terms - Natural convection, Rayleigh number, Prandtl number, Biot number.

\section{INTRODUCTION}

Given the number of practical applications, convective heat transfer in an enclosure has been studied extensively. The early investigations in this area include Ostrach [1], Wilkes and Churchill [2], and de Vahl Davis [3] who introduced a numerical solution for 2-D natural convection in a rectangular cavity. A large number of other studies followed examining a variety of cavities. Both the finite element and finite difference methods have been employed to solve the partial differential equations governing the buoyancy-driven flows. The former, while having the advantage of being adaptable to flow confined to odd geometries, lacks the simplicity of the latter. As a result, the majority of literature on numerical studies of natural convection is based on finite difference techniques, with most employing the SIMPLE (Semi-Implict Method for Pressure Linked Equations), an algorithm developed by Patankar [4] to resolve the coupling between velocity and pressure. Many numerical studies, for example, Rajkumar et al. [5], Martyushev and Sheremet [6], and Odewole and Edwards [7] selected to use the commercially available software package FLUENT with the solution option of SIMPLE. In the present study, a two-dimensional, time dependent numerical approach based on the projection or fractional step formulation method is applied to buoyancy-driven flow in a vertical slot with a narrow upper section as shown in Fig. 1. The fluid within this cavity is initially stagnant and at equilibrium with ambient. The left boundary is then subjected to a sudden increase in temperature while right boundary is

Manuscript received February 5, 2014; revised April 3, 2014.

E. Selamet is with the Center for Automotive Research The Ohio State University, Columbus, OH, 43210, USA (e-mail : selamet.2@ osu.edu).

A. Selamet is with the Department of Mechanical and Aerospace Engineering, The Ohio State University, Columbus, OH, 43210, USA (e-mail: selamet.1@osu.edu). allowed to transfer heat to ambient by convective cooling. The top and bottom surfaces are thermally insulated. An earlier work by Evren-Selamet et al. [8], studied natural convection in air within this type of cavity with sidewalls kept at different constant temperatures.

\section{FORMULATION}

In terms of the following dimensionless variables

$$
\begin{gathered}
x=x^{*} / L_{2}, \quad y=y^{*} / L_{2}, \\
u=u^{*} L_{2} / \alpha, \quad v=v^{*} L_{2} / \alpha, \\
t=t^{*} \alpha / L_{2}^{2}, \quad p=p^{*} L_{2}^{2} /\left(\rho \alpha^{2}\right), \\
\Theta=\left(T-T_{\infty}\right) /\left(T_{h}-T_{\infty}\right)
\end{gathered}
$$

the two-dimensional conservation equations for mass, momentum, and energy may be written for buoyancy-driven incompressible fluids as

$$
\begin{gathered}
\frac{\partial u}{\partial x}+\frac{\partial v}{\partial x}=0 \\
\frac{\partial u}{\partial t}+\frac{\partial u^{2}}{\partial x}+\frac{\partial u v}{\partial y}=-\frac{\partial p}{\partial x}+\operatorname{Pr} \nabla^{2} u \\
\frac{\partial v}{\partial t}+\frac{\partial u v}{\partial x}+\frac{\partial v^{2}}{\partial y}=-\frac{\partial p}{\partial y}+\operatorname{Pr} \nabla^{2} v+\mathcal{R} \operatorname{Pr} \Theta \\
\frac{\partial \Theta}{\partial t}+\frac{\partial u \Theta}{\partial x}+\frac{\partial v \Theta}{\partial y}=\nabla^{2} \Theta
\end{gathered}
$$

where $x^{*}, y^{*}$ are the coordinates $, u^{*}, v^{*}$ the velocities $t^{*}$ the time, $p^{*}$ the pressure, $T$ the temperature, $L_{2}$ the width of the lower section of enclosure, $\nabla^{2}$ the two-dimensional Laplacian, $\operatorname{Pr}=v / \alpha$ the Prandtl number (=6 in this study), $\mathcal{R}=$ $g \beta L_{2}^{3} \Delta T / v \alpha$, the Rayleigh number which accounts for the coupling between energy and momentum balances [9], [10]; $g$ being the gravitational acceleration, $\beta$ the coefficient of thermal expansion of the fluid, $\Delta T$ temperature difference between left boundary and ambient, $v$ the kinematic viscosity, and $\alpha$ the thermal diffusivity. The Boussinesq approximation is used for the density in buoyancy force.

\section{The Numerical SOLUTION PROCEDURE}

The unsteady Navier-Stokes equations, governing the flow under the Boussinesq approximation, are solved with primitive variables. Convective terms are discretized using a second-order Godunov method, an upwind based 
differencing approach, which has been shown to provide a robust discretization of the convective terms [11]-[13]. Diffusive terms are calculated with centered finite differences. The velocities, pressure gradients, and temperatures are defined at the grid points, and the pressure and divergence of the velocity are defined at the cell centers so that only temperature and velocity components are needed on the boundaries. The Godunov scheme is explicit and the time step is restricted by the Courant-Fredrichs-Lewy (CFL) condition. Convergence of the solution is checked by examining the variation of Nusselt number. The results shown here are obtained with the grid size of $\Delta x=1 / 43$ and $\Delta y=5 / 70$. Selecting a $\Delta x$ smaller than $\Delta y$ helps better resolve the boundary layer. For further details of the method applied in the present study, the reader is referred to Evren-Selamet [8], [14]. The same study has also discussed the validation of the algorithm developed for buoyancy-driven flows by comparing the results for a square cavity with the earlier study of de Vahl Davis [3], which serves as a benchmark on natural convection investigations.

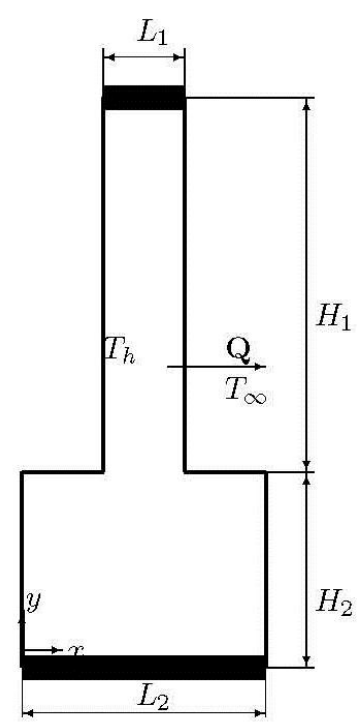

Fig. 1. Schematic of the system.

\section{A. Boundary Conditions}

Wall thickness is assumed negligible in this study. From heat balance at the right boundary $(r b)$ then

$$
-\left.k \frac{\partial T}{\partial x^{*}}\right|_{r b}=-h\left(T_{r b}-T_{\infty}\right)
$$

where $k$ is the thermal conductivity of water, $h$ the heat transfer coefficient between the wall and ambient, $T_{r b}$ temperature at right boundary, and $T_{\infty}$ the ambient temperature. With nondimensionalization of temperature, (5) becomes

$$
-\frac{\partial \Theta_{r b}}{\partial x}=B i \Theta_{r b}
$$

where $B i=h L / k$ is the Biot number. Due to infinitesimally thin wall, $B i$ here ends up coupling thermal conductivity of the fluid on one side of the wall with the heat transfer coefficient on the other, which is unconventional for this number. $\partial T / \partial y=0$ at the top and bottom walls since they are thermally insulated. Left wall is prescribed at a uniform temperature $T_{h}$. No-slip velocity conditions $(u, v=0)$ are employed on all walls.

\section{RESUlTS AND DISCUSSION}

Results presented here are obtained in a vertical slot with $H_{1}+H_{2}=5 L_{2}, L_{1}=L_{2} / 2$ and $H_{1} / H=5.5 / 7$ where $\mathrm{H}=\mathrm{H}_{1}+\mathrm{H}_{2}$. To examine the impact of $B i$ at different $\mathcal{R}$, four different $B i(=2,10,100$ and 1000) and three different $\mathcal{R}$ $\left(=10^{4}, 10^{5}\right.$ and $\left.3 \times 10^{5}\right)$ are considered. Examples of temperature distribution and stream function contours are shown in Figs. 2 and 3 for Grashof number $=5 \times 10^{4}$ and $B i=10$.

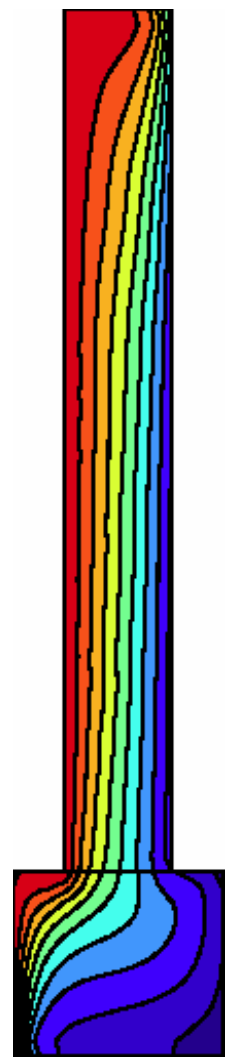

Fig. 2. Temperature contours (in increments of 0.1 ) for $G r=5 \times 10^{4}$, $P r=6$ and $B i=10$.

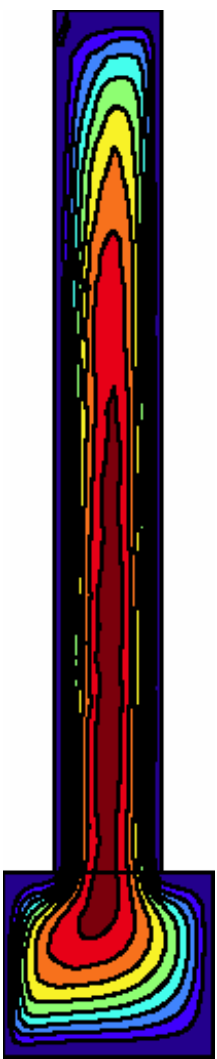

Fig. 3. Streamline contours (in increments of 1.0) for $\mathrm{Gr}=5 \times 10^{4}$, $P r=6$ and $B i=10$.
The predicted maximum velocity Vmax and average Nusselt number per unit length $\mathrm{Nu}$ on the sidewall are shown in Figs. 4 and 5, respectively, as a function of Biot number for different $\mathcal{R}$. The circles represent $\mathcal{R}=10^{4}$, squares $\mathcal{R}=10^{5}$, and filled circles $\mathcal{R}=3 \times 10^{5}$. The average Nusselt number is computed by integrating negative of temperature gradient in the fluid by trapezoidal rule over the right boundary. As evident from Figs. 4 and 5, Vmax and $N u$ increase asymptotically with Biot number. The increase becomes more pronounced at higher $\mathcal{R}$. When $\mathcal{R}$ is increased at constant $B i$, a larger temperature difference $\left(T_{h}-T_{\infty}\right)$ enhances the buoyancy forces which intensify the convective flow. Therefore, a more vigorous flow field is obtained with increasing $\mathcal{R}$ leading to higher $N u$ and $V \max$, as expected. 
Increasing $\mathrm{Bi}$ at low to mid range, for example, until about $B i=100$, leads to a stronger convective flow pattern enhancing the cooling of the cavity, whereas this effect tends to diminish at larger $B i$. Among the recent studies, for example, Martyushev and Sheremet [6] examined the variation of $\mathrm{Nu}$ and maximum velocity for different thermal conductivity ratio of wall/fluid in a cavity by considering convective and radiative heat transfer and observed similar behavior. They also noted similar trends with increasing Rayleigh number.

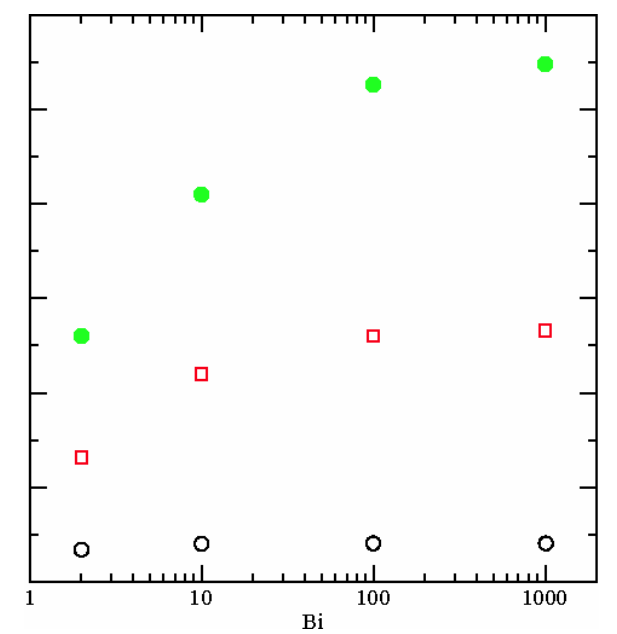

Fig. 4. Variation of maximum velocity ( $\operatorname{Vmax}$ ) with Biot number for $\mathcal{R}=10^{4}$ (circles), $10^{5}$ (squares) and $3 \times 10^{5}$ (filled circles).

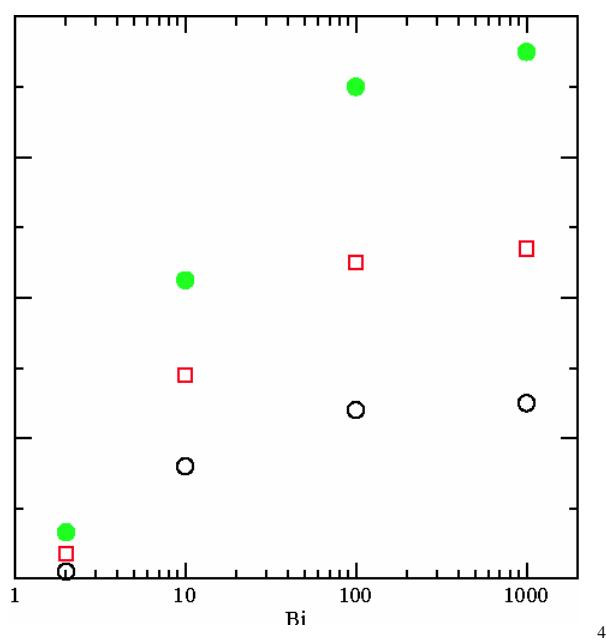

Fig. 5. Variation of Nusselt number with Biot number for $\mathcal{R}=10^{4}$ (circles), $10^{5}$ (squares) and $3 \times 10^{5}$ (filled circles).

\section{CONCLUSIONS}

Buoyancy-driven flow in a cavity with a narrow upper section subject to heating on one side and cooling on the other is numerically simulated by the projection method combined with a Godunov-type discretization for convective terms. The heat transfer is examined by varying $B i$ and $\mathcal{R}$. The results suggest that heat transfer increases asymptotically with increasing Biot number. The rate of this increase is a function of Rayleigh number. The predictions presented here are consistent with the available literature

\section{NOMENCLATURE}

gravitational acceleration
Gr $\quad \mathrm{g} \beta L_{2}^{3}\left(T_{h}-T_{\infty}\right) v^{2}$,

Grashof number

$H_{1}, H_{2}$ heights of narrow and wide sections of the cavity

$H_{1}+H_{2}$

$k$ thermal conductivity

$L_{1}, L_{2} \quad$ widths of the narrow and wide sections of the cavity

$\mathrm{Nu}$ average Nusselt number per unit length

$p \quad$ nondimensional pressure

$\operatorname{Pr} \quad v / \alpha$, Prandlt number

$\mathcal{R} \quad \mathrm{g} \beta L_{2}^{3}\left(T_{h}-T_{\infty}\right) / \nu \alpha$, Rayleigh number

$T$ temperature

$t \quad t^{*} \alpha / L_{2}$, nondimensional time

$u \quad u^{*} L_{2} / \boldsymbol{\alpha}$, nondimensional horizontal velocity

$v \quad v^{*} L_{2} / \boldsymbol{\alpha}$, nondimensional vertical velocity

$V_{\max } \quad$ nondimensional maximum velocity

$x, y \quad x^{*} / L_{2}, y^{*} / L_{2}$, nondimensional coordinates

\section{Greek letters:}

$\alpha \quad$ thermal diffusivity

$\beta \quad$ coefficient of thermal expansion

$\Theta \quad\left(T-T_{\infty}\right) /\left(T_{h}-T_{\infty}\right)$ nondimensional temperature

$\nu \quad$ kinematic viscosity

$\rho$ density

\section{Subscripts:}

$h \quad$ hot

$\infty \quad$ ambient

\section{REFERENCES}

[1] S. Ostrach, "Laminar natural convection flow and heat transfer of fluids with and without heat sources in channels with constant wall temperatures," NACA TN 2863, 1952.

[2] J. O. Wilkes and S. W. Churchill, "The finite difference computation of natural convection in a rectangular enclosure," AIChE J., 12, p. 161, 1966.

[3] G. de Vahl Davis, "Natural convection of air in a square cavity: A benchmark numerical solution," International Journal of Numerical Methods in Fluids, vol. 3, pp. 249-264, 1983.

[4] S. V. Patankar, Numerical Heat Transfer and Fluid Flow, Hemisphere, Washington, D.C., 1980.

[5] M. R. Rajkumar, G. Venugopal and S. Anil Lal, "Natural convection with surface radiation from a planar heat generating element mounted freely in a vertical channel," Heat Mass Transfer, vol. 47, pp. 789-805, 2011.

[6] S. G. Martyushev and M. A. Sheremet, "Conjugate natural convection combined with surface thermal radiation in an air filled cavity with internal heat source," International Journal of Thermal Sciences, vol. 76, pp. 51-67, 2014.

[7] A. Odewole and R. Edwards, "The characteristics of the velocity field in a slot-ventilated wall cavity," ARPN Journal of Engineering and Applied Sciences, vol. 6, no. 10, pp. 47-55, 2011.

[8] E. Evren-Selamet, V. S. Arpaci, and C. Borgnakke, "Simulation of laminar buoyancy-driven flows in an enclose," Numerical Heat Transfer, Part A, vol. 22, pp. 401-420, 1992.

[9] V. S. Arpaci and P. S. Larsen, Convective Heat Transfer, Prentice-Hall, Englewood Cliffs, NJ, 1984, ch. 2.

[10] V. S. Arpaci, A. Selamet, and S. Kao, Introduction to Heat Transfer, Prentice-Hall, Upper Saddle River, NJ, 2000, ch. 5.

[11] J. B. Bell, P. Colella, and H. M. Glaz, "A second order projection method for viscous, incompressible flow," in Proc. AIAA 8th Computational Fluid Dynamics Conf., Honolulu, Hawaii, 1987.

[12] J. B. Bell, H. M. Glaz, J. M. Solomon, and W. G. Szymczak, "Application of a second order projection method to the study of shear layers," in Proc. 11th International Conference on Numerical Methods in Fluid Dynamics, 1988.

[13] J. B. Bell, J. M. Solomon, and W. G. Szymczak, "A second order projection method for the incompressible Navier Stokes equations on quadrilateral grids," in Proc. the AIAA 9th Computational Fluid Dynamics Conference, Buffalo, NY, 1989. 
[14] E. Evren-Selamet, "Solution of Benard problem with the projection method," International Communications in Heat and Mass Transfer, vol. 22, pp. 59-70, 1995.

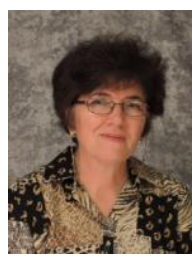

Emel Selamet received her Ph.D. and M.S. degrees in mechanical engineering, University of Michigan in 1991 and 1986 respectively. She got her M.Sc. degree in 1982 and B.Sc. degree in 1980 in mechanical engineering from Technical University of Istanbul. She is currently a research scientist at the Center for Automotive Research, The Ohio State University.
Ahmet Selamet received his Ph.D. in mechanical engineering and M.S. in aerospace engineering in 1989 and 1987 respectively from University of Michigan. And he obtained his M.Sc. degree in 1982 and B.Sc. degree in 1980 in mechanical engineering from Technical University of Istanbul. He is now working as a professor in the Department of Mechanical and Aerospace Engineering, The Ohio State University. 